\title{
Efficient Rendering of Strong Secondary Lighting in Photon Mapping Algorithm
}

\author{
Takehiro Tawara, Karol Myszkowski, and Hans-Peter Seidel \\ MPI Informatik Saarbruecken, Germany \\ \{tawara, karol, hpseidel\}@mpi-sb.mpg.de
}

\begin{abstract}
In this paper we propose an efficient algorithm for handling strong secondary light sources within the photon mapping framework. We introduce an additional photon map as an implicit representation of such light sources. At the rendering stage this map is used for explicit sampling of strong indirect lighting in a similar way as it is usually performed for primary light sources. Our technique works fully automatically, improves the computation performance, and leads to better image quality than traditional rendering approaches.
\end{abstract}

\section{Introduction}

In the rendering of production quality still images and animations, global illumination computations [7, 3] are usually performed using two-pass methods. In the first (preprocessing) pass, the lighting distribution over scene surfaces is sparsely computed using radiosity $[8,12,2]$ or photon mapping $[7,1]$ methods. In the second (rendering) pass more exact global illumination computation is performed on a per pixel basis using the results obtained in the first pass. To improve the spatial resolution of lighting details for a given camera view the so-called final gathering [9, 8, 12, 2] is commonly used. Usually the direct lighting is explicitly computed for each pixel, and the indirect lighting is obtained through the integration of incoming radiances, which is computationally expensive.

More efficient versions of this final gathering recently have been proposed specifically for hierarchical radiosity with clustering $[10,11]$. The final gathering costs also can be reduced by using the irradiance cache data structure $[14,15]$ which is more suitable for ray tracing methods. Within this method irradiance samples are lazily computed and sparsely cached in object space for a given camera position (a view-dependent process). The indirect illumination is interpolated for each pixel based on those cached irradiance values, which is significantly faster than the final gathering computation for each pixel. The irradiance cache tech- nique efficiently removes shading artifacts which are very difficult to avoid if the indirect lighting is directly reconstructed based on the radiosity mesh or the photon maps. However, the price to be paid for this high quality lighting reconstruction is long computation times, which are mostly caused by the irradiance integration that is performed for each cache location in the scene.

A naive sampling with uniform distribution of sampling directions in the space may lead to very poor convergence of the irradiance integration which manifests in noisy pixels for scenes with significant variations of lighting distribution. An efficient way to improve the performance of irradiance integration is to sample more densely those scene regions which significantly contribute to illumination at a given cache location. For glossy surfaces an easy importance sampling scheme can be considered by grouping sample directions around the reflection direction in respect to the eye position. However, for diffuse surfaces this importance criterion fails. In this work we propose an efficient importance sampling scheme which handles this difficult case. Our solution is embedded into the photon mapping algorithm framework [7]. In the following section we remind briefly basics of this algorithm.

\section{Photon Mapping}

The Photon Mapping [6, 7] works well even for complex environments, and can easily simulate all possible light paths. In particular, the method outperforms all other techniques in high quality rendering of caustic effects. The method consists of two passes: (1) lighting simulation through photon tracing and (2) rendering with the re-computation of direct lighting and view-dependent specular effects.

In the first stage of this method, photons are traced from light sources toward the scene and photon hit points are registered in a kd-tree structure, the so called photon map. This stage is very fast when compared to the second pass.

In traditional photon mapping algorithm photons are stored in the so-called caustic and global photon maps. The caustic photon map collects photons immediately after they 
are reflected or refracted by surfaces with specular light reflectance properties. The global photon map stores all photon hit points.

At the rendering stage caustic effects are reconstructed through a direct density estimation performed for photons stored in the caustic photon map. This enables the reconstruction of quickly changing lighting patterns which are typical for caustic effects. To obtain high quality caustics (reduce the stochastic noise without excessive blurring of caustic details) a huge number of photons are needed. This can be achieved by reinforcing shooting caustic photons in the direction of specular objects in the scene. This is an easy task for photons propagating directly from light sources to the specular surfaces. However, taking into account more complex photon paths involving collisions with diffuse or glossy surface before reaching the specular surfaces is still an open research issue.

Slowly changing (soft) indirect lighting is reconstructed based on the global photon map through the irradiance cache technique $[14,15,7]$. For each cache location, irradiance is integrated over the scene by sampling incoming energy for selected directions. Each sample involves a costly intersection computation performed by tracing a ray and the estimate of energy incoming from the point hit by the ray. The photon map is used for the incoming lighting reconstruction using the nearest neighbor density estimation method [4]. To reduce the density estimation cost Christensen [1] proposed to precompute irradiance and store the resulting values at sparsely selected photon locations on diffuse surfaces. When the final gather ray hits some object, a precomputed irradiance value for the nearest photon location is simply used. To reduce the variance of such sampling, the hemisphere of all possible directions is split into strata and a small number of sample directions (usually one) are randomly chosen for each stratum.

This works well for scenes with low variation of lighting distribution, but leads to enormous numbers of samples when density of photons in the global photon map significantly changes between scene regions. Ideally the angular density of samples should somehow correspond to the density of photons stored in the map. Estimating photon density would require projecting all those photons on the hemisphere centered at a given cache location, which is too costly. We propose a simpler solution which involves splitting photons in the global map between two maps. In the first map (we call it the high-energy photon map) photons in very bright scene regions are stored. The second map (we simply call it the low-energy photon map) stores the remaining photons, which effectively leads to small spatial variations of their density. In terms of irradiance integration this map can be properly sampled by a small number of uniformly distributed sampling directions. The high-energy map involves explicit directional sampling towards regions of high photon concentration with controllable angular density of irradiance samples. In the following section we introduce our algorithm for splitting the global photons between the two maps.

\section{Algorithm}

At the first step of our algorithm a voxel grid which contains the whole rendered scene is built. Each voxel has a counter which stores the number of photons hitting surfaces in this voxel. In the photon tracing stage, initially all photon hit points are stored in the low-energy photon map. However, when the number of photons stored in a given voxel is equal to a specified threshold value $c_{\max }$ all subsequent photons are stored in the high-energy photon map. $c_{\max }$ should be chosen so that photons are captured in this map only for strong secondary lights. In practice, the user decides the threshold irradiance value $E_{\max }$ which is then used to compute the corresponding $c_{\max }$ using the following relation:

$$
c_{\max }=\frac{E_{\max } A}{\Delta \Phi}
$$

where $\Delta \Phi$ is the radiant power carried by each photon and $A=\pi r^{2}$ is an estimate of the surface area in the voxel of the edge length $2 r . E_{\max }$ can be automatically selected as a function of the average scene irradiance $E_{a v g}$, which is estimated for a certain percentage $p_{\%}$ of the pilot photons as:

$$
E_{\text {avg }}=\frac{n \Delta \Phi}{A_{\text {total }}}
$$

where $n$ is the number of photon hit points for $p_{\%}$ of all traced photons (usually we assume $p_{\%}=10 \%$ ) and $A_{\text {total }}$ is an estimate of surface area for all objects in the scene.

Figure 1 shows the distribution of photons stored the high-energy photon map, which was obtained using this procedure. Although this scheme is fast and sufficiently accurate for our purposes, more elaborate methods to examine the density of photons are presented in [13].

In the rendering stage, the high-energy photon map is used to explicitly sample irradiance at given point $x$ in the scene by shooting rays toward the random locations inside the corresponding voxels. The number of rays per voxel can be proportional to the photon count in each voxel or it can be just a fixed number. The reflected radiance at the location $x$ for strong secondary light sources is represented as the integral of the differential irradiance $d E$ for each high energy voxel:

$$
L(x, \omega)=\int_{\Omega} f\left(x, \omega, \omega^{\prime}\right) V\left(x, \omega^{\prime}\right) d E\left(x, \omega^{\prime}\right)
$$

Here $\Omega$ is the set of high energy voxels and $V\left(x, \omega^{\prime}\right)$ is the visibility function $\left(V\left(x, \omega^{\prime}\right)=1\right.$ when the ray traced from 


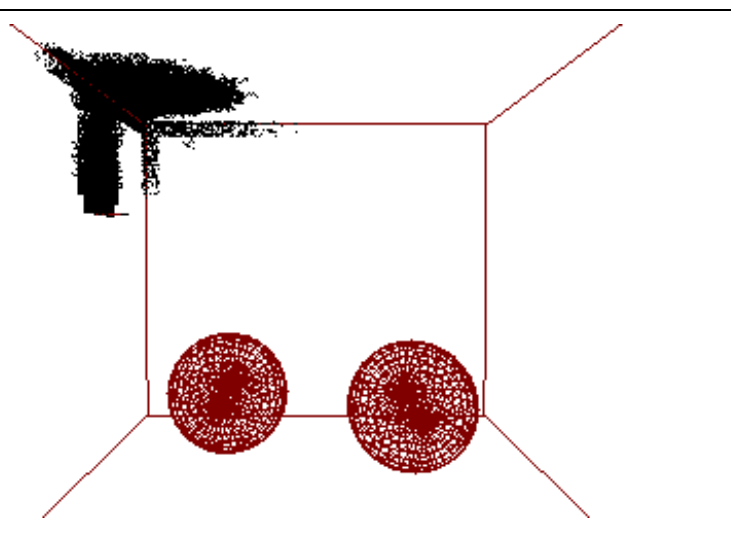

Figure 1. Distribution of photon hit points stored in the high-energy photon map for the scene shown in Figure 3. The black dots in the upper left region around the primary light source represent photons from this map.

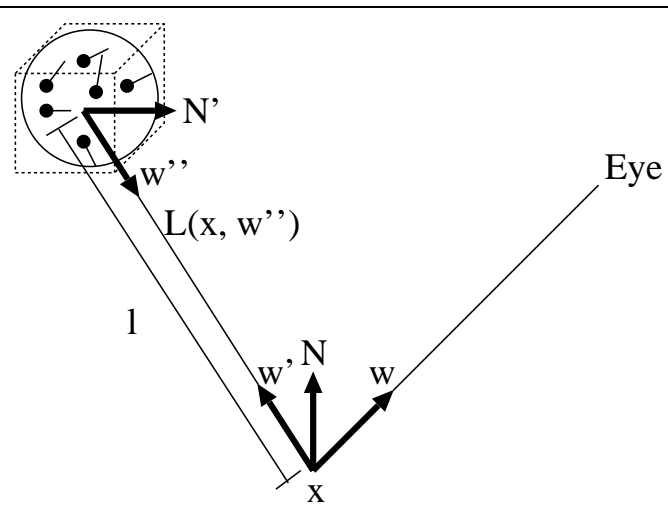

Figure 2. The schematic view of computing the differential irradiance $d E$ for the selected voxel. The black dots inside the voxel represent photon hit locations with marked incoming directions.

$x$ in the direction $\omega^{\prime}$ arrives to the selected voxel, otherwise $\left.V\left(x, \omega^{\prime}\right)=0\right)$.

Figure 2 illustrates the schematic view of the differential irradiance $d E$ coming from a selected voxel. The differential irradiance $d E$ is computed as:

$$
\begin{aligned}
d E\left(x, \omega^{\prime}\right) & =L_{i}\left(x, \omega^{\prime \prime}\right)\left(\omega^{\prime} \cdot N\right) d \omega^{\prime} \\
d \omega^{\prime} & =\frac{\left(\omega^{\prime \prime} \cdot N^{\prime}\right) A}{l^{2}}
\end{aligned}
$$

The reflected radiance $L_{i}\left(x, \omega^{\prime}\right)$ from a secondary light source (a selected voxel) is approximated by the density estimation of the high-energy photon map. $N$ and $N^{\prime}$ are the normal vectors at the location $x$ and the selected location inside the voxel, respectively. Here, $\omega^{\prime \prime}=-\omega^{\prime}$ and $l$ is the distance between the location $x$ and the location inside the voxel. The meaning of $A$ is the same as in equation (1).

\section{Results}

We tested our algorithm for scenes shown in Figures 3 and 4. All images are rendered on a Pentium 4 Xeon 1.7 $\mathrm{GHz}$, Debian GNU/Linux PC.

For images in Figure 3 the irradiance integration is performed for each pixel (the final gathering procedure). Figure 3 a presents the result of stratified sampling for 768 samples per pixel using the traditional global photon map. Although rendering requires 21 minutes stochastic noise is still perceivable. Figure $3 \mathrm{~b}$ shows the results obtained using our technique. The image is rendered using 48 stratified samples towards the low-energy photon map and 230 explicit samples towards high-energy secondary light sources. The rendering time amounts to 9 minutes. Although in our approach nearly three times less samples have been computed the overall image quality is significantly better than in Figure 3a.

In Figure 4, we demonstrate our algorithm in the more complex scene. The image is rendered using the irradiance caching. Because the irradiance integration is performed only on the irradiance cache locations, significant speedup of the computation is achieved. 300 stratified samples towards the low-energy photon map and 98 explicit samples towards the high-energy photon map are used for each irradiance integration. The total number of cache locations is 13,666 and the rendering time is 10 minutes for the image resolution of $1,128 \times 480$ pixels.

\section{Discussion}

Our algorithm separates the global photon map into the low- and high-energy photon maps. The former map is used for the irradiance integration as in traditional photon mapping algorithm. Because of the lower variation of photon distribution in this map a high accuracy of such integration can be easily achieved using only a small number of sampling directions. The high-energy photon map associated with the voxel grid identifies strong secondary light sources which are explicitly sampled by shooting rays toward the corresponding directions.

A similar idea is implemented in the Radiance rendering system [16], in which bright scene objects can be manually chosen as the secondary light sources. This scheme works only in the case when the bright objects are known in advance and can be manually selected as virtual lights to be explicitly sampled at the rendering stage. The advantage of our algorithm is that it can be applied automatically 

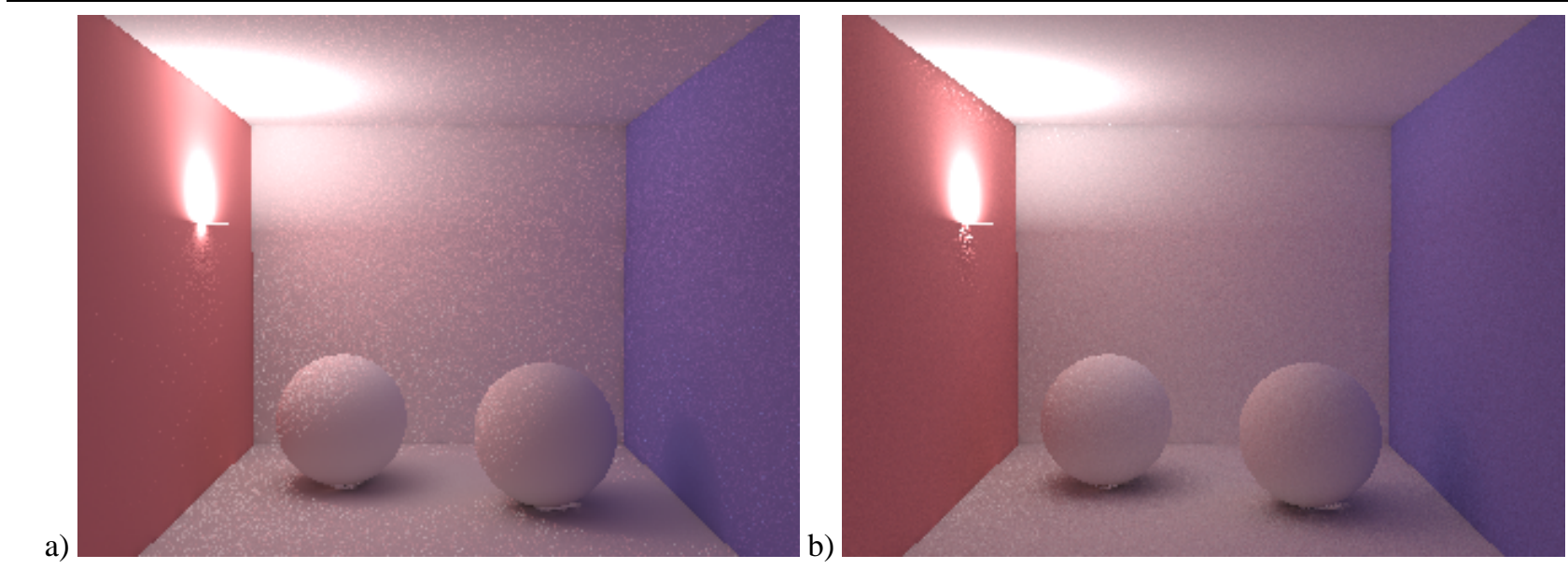

Figure 3. Final gathering procedure. Size: $320 \times 240$ pixels, a) 768 stratified samples/pixel, rendering time $21 \mathrm{~min}$ and b) 278 (48 stratified samples +230 explicit samples) samples/pixel, rendering time 9 $\min$.

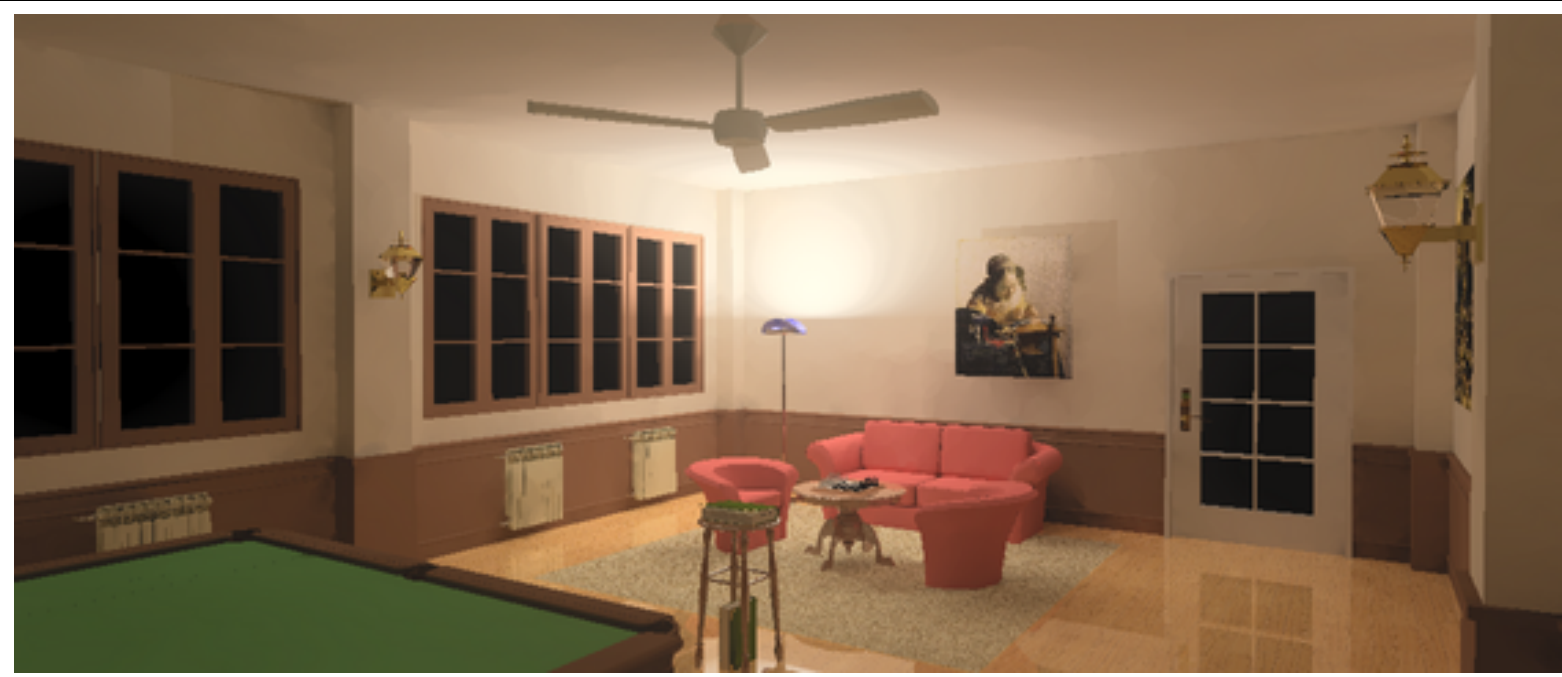

Figure 4. The image is rendered by our algorithm using the irradiance caching. Size: $1,128 \times 480$ pixels, 398 (300 stratified samples + 98 explicit samples) samples/cache, 13,666 caches, rendering time $10 \mathrm{~min}$.

for any scene. Since we use the photon map and voxel grid data structures, our secondary light source representation is independent of any type of scene geometry. In our technique strong secondary light sources are automatically selected and the user must just specify one parameter to control this choice.

Another related work is the importance sampling using the photon map algorithm [5] in which the importance directional function is built based on the photon map at each rendered pixel. Our algorithm requires to build the impor- tance positional function only once as the photon count in the voxel grid. In our method, the important directions are immediately found for each pixel by picking the selected voxels.

\section{Conclusions}

We presented an efficient algorithm for rendering scenes with strong secondary light sources. The performance of our algorithm is the better the more variation in secondary light- 
ing is present in the scene. Our technique can be considered as a variant of importance sampling embedded into the framework of photon mapping algorithm.

As future work we plan to extend our technique to handle dynamic scenes. We intent to exploit the temporal coherence in indirect lighting distribution to improve the computation efficiency.

\section{References}

[1] P. Christensen. Photon mapping tricks. In Siggraph 2002, Course Notes No. 43, A Practical Guide to Global Illumination using Photon Mapping organized by Jensen, $H$.W., pages 93-121, 2002.

[2] P. Christensen, D. Lischinski, E. Stollnitz, and D. Salesin. Clustering for glossy global illumination. ACM Transactions on Graphics, 16(1):3-33, 1997.

[3] P. Dutre, P. Bekaert, and K. Bala. Advanced Global Illumination. A. K. Peters, Natick, MA, 2003.

[4] P. Heckbert. Adaptive radiosity textures for bidirectional ray tracing. In Computer Graphics (ACM SIGGRAPH '90 Proceedings), pages 145-154, August 1990.

[5] H. Jensen. Importance driven path tracing using the photon map. In Eurographics Workshop on Rendering, pages 326$335,1995$.

[6] H. Jensen. Global illumination using photon maps. In Eurographics Workshop on Rendering, pages 21-30, 1996.

[7] H. Jensen. Realistic Image Synthesis Using Photon Mapping. A. K. Peters, Natick, MA, 2001.

[8] D. Lischinski, F. Tampieri, and D. P. Greenberg. Combining hierarchical radiosity and discontinuity meshing. In Proc. of Siggraph'93, pages 199-208, 1993.

[9] M. Reichert. A Two-Pass Radiosity Method to Transmitting and Specularly Reflecting Surfaces. M.Sc. thesis, Cornell University, 1992.

[10] A. Scheel, M. Stamminger, and H.-P. Seidel. Thrifty final gather for radiosity. In Rendering Techniques 2001: 12th Eurographics Workshop on Rendering, pages 1-12. Eurographics, June 2001.

[11] A. Scheel, M. Stamminger, and H.-P. Seidel. Grid based final gather for radiosity on complex clustered scenes. In $E u$ rographics, 2002.

[12] B. Smits. Efficient Hierarchical Radiosity in Complex Environments. Ph.D. thesis, Cornell University, 1994.

[13] F. Suykens and Y. Willens. Density control for photon maps. In Rendering Techniques 2000: 11th Eurographics Workshop on Rendering, pages 23-34, June 2000.

[14] G. Ward, R. F., and R. Clear. A ray tracing solution to diffuse interreflection. In Computer Graphics (SIGGRAPH 88 Conference Proceedings), pages 85-92, 1988.

[15] G. Ward and P. Heckbert. Irradiance gradients. In Eurographics Workshop on Rendering, pages 85-98, 1992.

[16] G. Ward and R. Shakespeare. Rendering with Radiance. Morgan Kaufmann, Los Altos, California, 1998. 